\title{
Corona controversies
}

(BIRDEM Med J 2020; 10(3): 141-144)

\section{Origin of the virus}

There was dispute over where from the corona virus came.

\section{Animal source}

The first outbreak was in Wuhan City of Hubei Province, China in workers of wet live animal market. The speculation was that, bat is the source, through an intermediate civet (cat like animal), it spread to human. The researchers analyzed partial sequences of some 1240 corona viruses found in bats of China. They reported that the virus fuelling the pandemic, severe acute respiratory syndrome is corona virus 2 (SARS$\mathrm{CoV}-2$ ) and is closely related to a group of virus found in bats (Rhinolophus) and finally concluded that SARS$\mathrm{CoV}-2$ is a chimer of snake and bat gene.

\section{Laboratory source}

A dispute over lab escape of virus was there, because the first outbreak occurred in the heart of the city where Wuhan Institute of Virology (WIV) is situated. The WIV has the highest supervised biosafety (BSL-4). Scientists believe that probe into the matter of lab escape is in many ways hypocritical.

\section{A product of nature}

Scientists all over the world studied virus's genomic detail and concluded that it emerged naturally rather than from a laboratory. They also studied the receptor binding domain (RBD) and furin cleavage site in human tissue which further prove uniqueness of the virus. So, it is a novel corona virus and was named as the severe acute respiratory syndrome coronavirus-2 (SARS-CoV2, 2019-nCoV). The disease it causes is corona virus disease-19 (COVID-19).

\section{Transmission}

Experts believe the virus that causes COVID-19 spreads mainly from person to person.

\section{Droplets or aerosols/air borne}

Anyone who is within 6 feet of infected person can breathe it into their lungs. Lastly research shows that the virus can live in the air for up to 3 hours, so airborne transmission is possible.

\section{Fomite/feco-oral}

Bad personal habit helps fomite transmission, may also feco-oral transmission.

\section{Vertical}

There are records of vertical transmission.

\section{Source/nidus}

The virus most often spreads through people who have symptoms. However, spread from asymptomatic and pre-symptomatic people is possible. A person can transmit 3-5 days before he/she becomes symptomatic and 2 weeks after recovery from the disease. Transmission of COVID-19 is exponential, whereas flu transmission is static ie one COVID person will transmit it to many but one flu will transmit to one.

\section{Signs and symptoms}

COVID-19 patients may be milder (ILI, 80\%), moderate (pneumonia), severe (severe pneumonia) and critical (5\%) (ARDS, septicemia and hypoxic respiratory failure). COVID-19 is a multisystem viral disease, may have superadded infection and may induce inflammation. Common symptoms of COVID-19 are fever, tiredness and dry cough; the beginning symptoms are similar to the common cold. In 3 days, the patient's throat starts to feel a bit painful, body temperature begins rising and in 5-6 days it goes higher with distressing cough. Around 1 in every 5 people who are infected with COVID-19 develop difficulty in breathing and require hospital care. Researchers in China found that the most common symptoms among people who were hospitalized with COVID-19 include: fever (99\%), fatigue (70\%), dry cough (59\%), loss of appetite (40\%), body aches (35\%), shortness of breath (31\%), mucus or phlegm (27\%). CDC guideline suggested screening in COVID-time to go to direct treatment avoiding RTPCR to cope with scarcity of kits. 


\section{Special forms}

a) Vulnerable group: elderly (more than 60 years), pregnant, chronic kidney disease, chronic liver disease, obstructive air way disease, heart failure, etc

b) Atypical form: COVID with stroke/thromboembolism/ myocarditis

c) COVID-19 with sequlae like Aspergillous pneumonia

\section{Pathophysiology}

Eighty percent are mild disease described as influenza like illness (ILI), remaining are severe acute respiratory illness (SARI). The corona virus has 4 structural proteins viz spike protein, membrane protein, envelope protein and nucleocapsid protein. There may be overlap buty two basic steps happen in infection 1. Virus entry: The virus enters the cell via ACE 2 receptors. It can involve any organ but predominantly respiratory system. In severe disease it attacks CVS and renal system. The virus fuses with receptor, enter the cell and multiply and 2. Body response: Normally innate immune system with the help of macrophages, neutrophils and natural killer cells (NK cells) causes viral destruction and elimination. The adaptive immune system, with the help of cytotoxic T lymphocytes (CTLs) and specific antibodies from plasma cells, causes viral destruction and elimination.

\section{Severe diseases}

In these groups of patients antibody response is inadequate, NK cells and CTLs fails to clear the damaged cells and fails to inhibit macrophage activity. There is severe inflammatory response with release of inflammatory cytokines specially IL 6 . There is recruitments of neutrophil and activation of macrophages and destruction of these cells produces cascade of events. In the lungs there is alveolar edema, interstitial edema, decrease of surfactant and alveolar collapse, so there is ARDS and hypoxic respiratory failure.

\section{High temperature}

IL-1, IL-6, TNF- $\alpha$ causes release of PGE-2 in hypothalamus and that resets the thermostat and increase the body temperature giving rise to high fever.

\section{Renin angiotensin system (RAS)}

It was presumed that use of ACE-i/ARB leading to increased expression of ACE2 could potentially facilitate infection with COVID-19. Clinical evidences and statements by ESC, ACC and WHO, nullified this.

\section{Cytokine release syndrome (CRS)}

CRS like syndrome (cytokine storm)/macrophage activation syndrome/hemophagocytic lymph histiocytosis- is characterized by oxygen saturation $<$ $93 \%$ on room air. Pao $2 / F i o 2<300 \mathrm{~mm} \mathrm{Hg}$. Respiratory rate $>25$ breaths/minute or respiratory distress. D-dimer $>1000 \mathrm{ng} / \mathrm{ml}, \mathrm{CRP}>100 \mathrm{mg} / \mathrm{L}, \mathrm{LDH}>245$ units/L, ferritin $>500 \mathrm{mcg} / \mathrm{L}$, decrease in absolute lymphocyte count $<800 /$ microL.

\section{Hypercoagulable state}

In vascular endothelium, there are ACE2 receptors. Direct inflammation of endothelium causes release of procoagulants (VWF), aVIII. Cytokine storm by activating procoagulants and inhibiting anticoagulants causes hypercoagulable state. As a result, there is widespread thromboembolic complication in both pulmonary and systemic circulation.

\section{Cardiovascular system}

Besides T2 pneumocytes, ACE2 is predominantly expressed in the endothelium of coronary and renal vessels. Direct viral invasion of heart, hypoxia, cytokine storm specially IL6, sympathetic hyperactivity all of these can cause myocardial injury with type 2MI, heart failure, tachyarrhythmias, carditis and even sudden death.

\section{Investigation of COVID-19 cases}

Routine test may be abnormal and informative in initial days. Total count may be very high without superadded infection. Very high neutrophil with low lymphocyte is characteristic. Though viral infection, ESR often is very high; so is CRP. Feritin, LDH, D-dimer, LDH should routinely be tested in suspected cases.

Antigen test: So far there is no antigen (kit test) except the one used in Tiwan with nucleocapsid protein with low sensitivity $(<40 \%)$.

Antibody: Antibody tests has been approved by FDA in recent days. It's best use is for surveillance, not for diagnosis.

Imaging: Both $\mathrm{HRCT}$ and chest $\mathrm{x}$-ray show characteristic lung shadowing and very much 
informative even at 5-6 days of infection and even in asymptomatic person and also with RT-PCR negative cases. HRCT, in fact, is as sensitive as RT-PCR; though to confirm a case one need positive RT-PCR.

RT-PCR for corona: The costly sequential complex method is needed to confirm cases. The false negativity and enormous false positivity is annoying.

\section{Treatment}

\section{Non-pharmacological treatment}

General care like warm gurgling, warm water. lemon water drinking, vitamins, minerals, herbals etc. has created tremendous attraction by people and media. May have some symptomatic relief but should not be taken as remedy or prevention of COVID.

\section{Non-conventional pharmacotherapy}

The anti-helminthic Ivermectin(discoverer awarded Nobel prize 2015), antibiotic doxycycline, azithromycin, anti-inflammatory hydroxychloroquine have become popular for their non-specific anti-RNA actions, antiinflammatory action; these actions mostly are at site of virus fusion to cell surfaces in vivo tests. These drugs have been used by physicians and institutions randomly in last few months. There are publications which were not peer reviewed. So far, there is no randomized controlled trial, mostly of individual and real life trial basis. Many publications have been withdrawn, lacking authentications.

\section{Pharmacotherapy}

Antiviral drug: Favipiravir and Remdesivir, two broad spectrum anti-RNA drugs, are being used in recent days in our country. Favipiravir is for Fdexamethasonelu and remdisivir worked against Ebola. Many other antivirals were also tried.

Antibiotic: Broad spectrum antibiotic (most cases parenteral) along with second one covering atypical infection is being used. Azythromycin though not better than clarithromycin is of choice because of its better lung penetration and proven prolonged therapy.

Corticosteroids: RECOVERY trial has shown benefits of $6 \mathrm{mg}$ dexamethasone beneficial for patients needing intervention oxygen therapy. Both methyl prednisolone $(1 \mathrm{mg} / \mathrm{kg})$ and dexamethasone of equivalent dose have been shown to be beneficial for short time benefit. Only thing, sudden or rapid withdrawal can cause clinical or biochemical rebound effect.
Anticoagulant: Italian post-mortem study has proved the anticoagulants are essential therapy for severe cases. Enoxaparin 60 is the therapeutic dose, $40 \mathrm{mg}$ can be used as prophylaxis and in case of normal or low Ddimer and patients with eGFR 30 or less. Frequent injection hence fear of exposure is hindrance to heparin. Both heparin and enoxaparin has added antiinflammatory action which give them superiority over NOAC. NOAC can be used in follow up cases. Those having evidence of thromboembolic phenomenon need follow up anticoagulants.

Immunotherapy: IVIg and hyper immune plasma therapy is being used in many canters;plasma therapy received nobel prize in 1901 for diphtheria treatment.

Interferon beta inhalers have also been used in UK.

Anti-inflammatory drugs: Tocilizumab and other biologics against interleukins and JAK receptors are being used in varied ways.

Oxygen therapy: There is a term happy hypoxia which is peculiar in COVID pneumonia. Patient seems to have no respiratory distress but oximetry shows SPO2 less demanding oxygen inhalation.

\section{All the drugs are of off level}

Remdesivir has been recommended as emergency use authorization (EUA), antivirals has been advocated lastly for 5 days use. Apparently only treatment essential and most effective for COVID pneumonia is oxygen inhalation. Oxygen should be used with all effort with as much concentration as possible aiming to avoid ventilation.

All these drugs have been included in different guidelines, which has been introduced in COVID era with modification by medical authorities subject to available knowledge and experiences. One thing is established every one is beneficial if used in proper time for proper patients.

\section{Prevention}

\section{Self-protection}

After 8 months of the pandemic it has become established that personal protection is the best way. Selfdistancing, proper hand washing and effective use of facemask can save a person, a family, a community and the population. 


\section{Community protection}

Quarantine, if someone is exposed and isolation if someone is diseased, imposes burden on economy but have to be followed.

\section{Vaccines}

AN effective COVID vaccine at this time is most wanted thing for mankind. Sino pharma of China, Bion tech of Germany, Moderna of USA and Oxford London are destined to give their vaccines to the market before the end of this year 2020. Russia already has introduced their vaccine and approved for marketing.

\section{Conclusion}

Corona virus has shattered the economy of whole world, autonomy of mankind. Health system of almost all nations has been proved ineffective to deal with it. Ever changing knowledge of pathology, human effect and treatment modalities are paving the way for extensive research and knowledge hunting. 